\title{
ON ROUGHNESS MEASUREMENT BY ANGULAR SPECKLE CORRELATION
}

\author{
F. Salazar ${ }^{1)}$, T. Belenguer ${ }^{2)}$, J. García ${ }^{1)}$, G. $\operatorname{Ramos}^{2)}$ \\ 1) Department of Applied Physics (FARN), ETSI Minas (UPM), C/Rios Rosas 21, 28003 Madrid, Spain (هfelixjose.salazar@upm.es) \\ 2) LINES-INTA. Department of Space and Science of Electronic Technologies, Carretera de Ajalvir km 4.5. 28850 Torrejón de Ardoz. \\ Madrid, Spain
}

\begin{abstract}
In this work, the influence of both characteristics of the lens and misalignment of the incident beams on roughness measurement is presented. To investigate how the focal length and diameter affect the degree of correlation between the speckle patterns, a set of experiments with different lenses is performed. On the other hand, the roughness when the beams separated by an amount are non-coincident at the same point on the sample is measured. To conclude the study, the uncertainty of the method is calculated.
\end{abstract}

Keywords: roughness, speckle, non-destructive testing.

(C) 2012 Polish Academy of Sciences. All rights reserved

\section{Introduction}

In different fields of the industry, the texture and finishing of a surface are of great importance for many applications [1-3]. For instance, the capacity of wear, lubrication, resistance to fatigue and external aspect of a material, are examples in which the state of the surface represents an important role. Industrial batch production requires that all the pieces have the same characteristics. The products should satisfy not only functional requirements, but also security and quality requirements. When anomalies in the production appear, roughness is one of the most variable parameters, and that is a reason to develop methods to measure it.

There are many methods for measuring roughness $[4,5]$. The most conventional technique is the tactile profilometer, which is based on the registration of the microscopic surface heights by means of a stylus. The statistical processing of the data allows the determination of parameters such as $R_{\mathrm{a}}$ and $R_{\mathrm{q}}$. This method has proved to be very useful; however, it requires scanning the surface under examination with the consequent consumption of time. Other disadvantages of this procedure are the impossibility to use it in soft surfaces in which the contact pressure may damage the surface or in machinery where the pieces must be examined in-situ. In this context, optical methods can play an important role, because they are contactfree and fast. Among them, optical techniques based on the speckle phenomenon have been used in the least twenty years due to their advantages.

The different procedures based on the speckle phenomenon for roughness measurement and their applications are well known [6-14]. This article deals with the classical angular speckle correlation technique (ASC), which requires an accurate alignment of the optical system and which is very sensitive to environment vibrations. Recently, a new arrangement of ASC without lens has been used to determine the roughness of surfaces [15, 16]. Despite the simplicity of this practical approach, it is beyond the scope of the present investigation. 
In this paper we try to study in detail two experimental questions that should be taken into account when the ASC method is employed. Firstly, we analyze the influence of the characteristics of the lens (focal length and diameter) on the degree of correlation of two speckle patterns and, secondly, we investigate how an imperfect coincidence of the two incident beams at the same point on the sample affects the correlation degree.

\section{Theory}

As it is well known, the angular speckle correlation technique is based on calculating the correlation between two speckle patterns. The first one is obtained by directing a coherent beam onto the rough sample under an angle $\alpha$, and is referred to as the first exposition; the second pattern is obtained in the same way, but the incidence angle of the beam is angularly displaced by a quantity $\delta \alpha$.

Assuming a Gaussian distribution for the surface heights, the correlation of the speckle fields for two angles in the Fourier plane is [17]:

$$
C=\exp \left[-\left(\left(\sin \alpha_{i} \delta \alpha+\sin \alpha_{o} \frac{\Delta \xi}{f}\right) k \sigma_{h}\right)^{2}\right] \exp \left[-\left(\frac{L k}{2 f}\right)^{2}\left(\left(\Delta \xi-\frac{\cos \alpha_{i}}{\cos \alpha_{0}} f \delta \alpha\right)^{2}+(\Delta \eta)^{2}\right)\right]
$$

where $\sigma_{h}$ is the variance of the surface, $k$ the wave number of the monochromatic radiation, $L$ is the half-width of the beam, $f$ the focal length of the lens, and $\alpha_{i}$ and $\alpha_{o}$ the angles of incidence and observation, respectively. $\xi$ and $\eta$ refer to the coordinate system on the CCD array (Fourier plane). In order to obtain a roughness-dependent correlation, the following conditions must be fulfilled:

$$
\Delta \xi-\frac{\cos \alpha_{i}}{\cos \alpha_{0}} f \delta \alpha=0
$$

and

$$
\Delta \eta=0
$$

In the case of our investigation, $\alpha_{i}=\alpha_{o}$, then Eq. (2) becomes

$$
\Delta \xi=f \delta \alpha
$$

and the correlation

$$
C=\exp \left(-\left(2 k \sin \alpha_{i} \delta \alpha \sigma_{h}\right)^{2}\right)
$$

which depends directly on $\sigma_{h}$. Eqs. (3) and (4) show that the speckle pattern shifts by an amount $f \delta \alpha$ as a consequence of the change of the incidence angle. This means that we must move the position of the camera to obtain a maximum correlation of the two speckle fields. 


\section{Experimental results}

\subsection{Influence of the lens}

Fig. 1 shows the optical arrangement employed for the measurements. A sample of titanium used for aerospace applications was tested. A laser LS is directed to a beam splitter which divides the radiation in two parts. The first one (b1) passes through a variable reflective mirror and strikes on the sample. The second (b2) is reflected in the mirror M impacting on S. A part of the light scattered by the surface is collected by the lens and focused on the CCD array. The mirror GM has been tuned in both expositions to obtain the same intensity on the camera, taking advantage of the dynamic range and avoiding errors after processing.
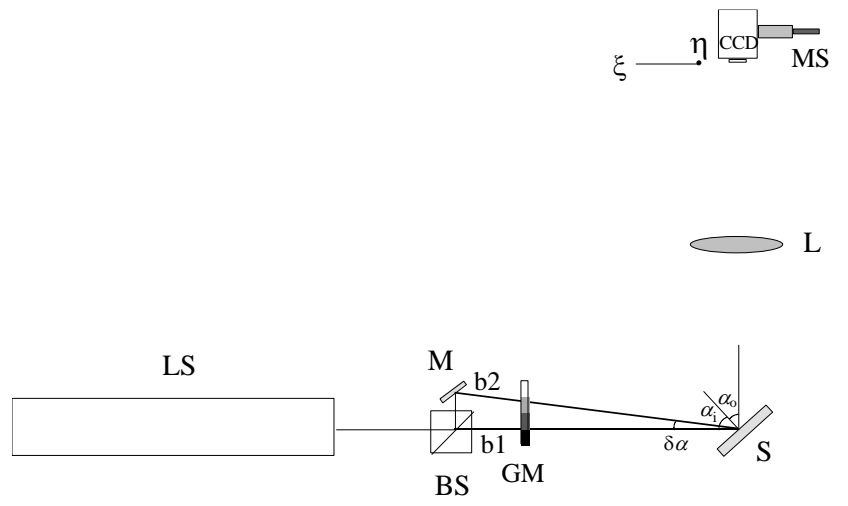

Fig. 1. Experimental set-up. LS, laser beam; BS, Beam splitter; M, mirror; GM, gradual mirror; $\alpha_{i}$, incidence angle; $\alpha_{o}$, observation angle; S sample; L, lens; MS, micrometer screw; CCD camera.

With the aim to determine the influence of the characteristics of the optical system, 13 lenses of different diameters and effective focal lengths were chosen. For all experiments $\lambda=632.8 \mathrm{~nm}, \alpha_{i}=\alpha_{o}=45^{\circ}$, and $\delta \alpha=1.9^{\circ}$. The experimental results obtained for the correlation coefficient $C$ and the roughness $\sigma_{h}$ are represented in Table 1. For a given value of $C$, the roughness may be directly calculated by means of the curve of Fig. 2. As can be observed, $C$ varies from 0.8359 to 0.9191 . For a given diameter, the value of the correlation, and thus the roughness, varies slightly as $f$ grown, but for a particular focal length, $C$ diminishes when $D$ becomes higher $(f=16 \mathrm{~cm}$ and $f=26 \mathrm{~cm})$. For diameters greater than 8 $\mathrm{cm}$, the tendency is a decreasing correlation.

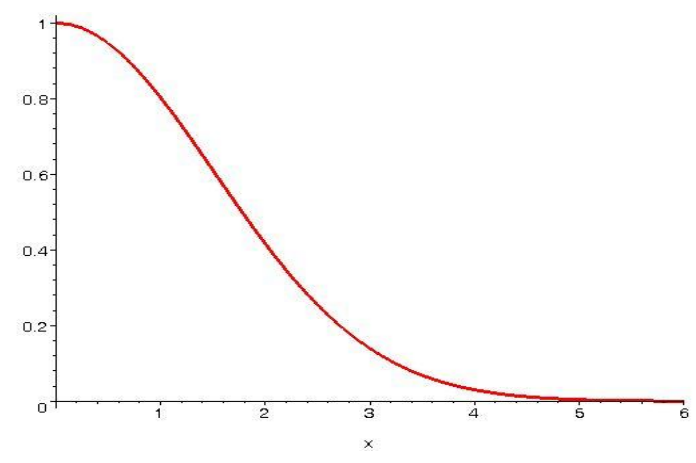

Fig. 2. Speckle correlation coefficient $C$ as function of $R_{\mathrm{q}}$ for $\lambda=632.8 \mathrm{~nm}, \alpha_{i}=\alpha_{o}=45^{\circ}$, and $\delta \alpha=1.9^{\circ}$. 
Table 1. Experimental results. The two numbers located in each box correspond to the correlation coefficient $(C)$ and roughness $\left(\sigma_{h}\right)$, respectively.

\begin{tabular}{|c|c|c|c|c|c|c|c|c|}
\hline $\mathrm{D}(\mathrm{cm})$ & 4.3 & 5 & 6 & 7.3 & 8 & 10 & 13 & 19.5 \\
\hline $\begin{array}{l}\mathrm{f}=5 \mathrm{~cm} \\
\sigma_{h}(\mu \mathrm{m})\end{array}$ & $\begin{array}{l}0.9076 \\
0.67\end{array}$ & & & & & & & \\
\hline $\mathrm{f}=5.5 \mathrm{~cm}$ & & $\begin{array}{l}0.8866 \\
0.73\end{array}$ & & & & & & \\
\hline $\mathrm{f}=8.5 \mathrm{~cm}$ & & $\begin{array}{l}0.9011 \\
0.69\end{array}$ & & & & & & \\
\hline $\mathrm{f}=10 \mathrm{~cm}$ & $\begin{array}{l}0.8904 \\
0.73\end{array}$ & $\begin{array}{l}0.9191 \\
0.62\end{array}$ & & & & & & \\
\hline $\mathrm{f}=12.5 \mathrm{~cm}$ & & & $\begin{array}{l}0.9004 \\
0.69\end{array}$ & & & & & \\
\hline $\mathrm{f}=15 \mathrm{~cm}$ & & & & $\begin{array}{l}0.8915 \\
0.72\end{array}$ & & & & \\
\hline$f=16 \mathrm{~cm}$ & $\begin{array}{l}0.8869 \\
0.73\end{array}$ & & & & $\begin{array}{l}0.8405 \\
0.89\end{array}$ & & & \\
\hline $\mathrm{f}=26 \mathrm{~cm}$ & $\begin{array}{l}0.9086 \\
0.66\end{array}$ & & & & & \begin{tabular}{l|}
0.8368 \\
0.90
\end{tabular} & & \\
\hline $\mathrm{f}=30 \mathrm{~cm}$ & & & & & & & $\begin{array}{l}0.8565 \\
0.84\end{array}$ & \\
\hline$f=38 \mathrm{~cm}$ & & & & & & & & $\begin{array}{l}0.8359 \\
0.91\end{array}$ \\
\hline
\end{tabular}

A problem arises when we have to decide what set of lenses is more adequate for roughness measurement using an optical set-up. In principle, with only the data shown in Table 1 , there is no easy answer to this question.

To investigate this difficulty, a test with a confocal microscope was performed, so that we can compare the obtained results with those found through the speckle technique.

Confocal microscopy means that the illumination point source and the collecting system are focused at the same time and in the same point. This idea has been widely used to develop confocal profilers that measure the surface height from smooth to very rough surfaces. Normally, the sample is scanned vertically in many steps, so that every point on the surface passes through a pinhole to the focus plane. The height of the surface at each pixel location on the CCD system is found by collecting the peak of the narrow axial response. The final confocal image is formed by the accumulation of different acquisition planes during the scanning process. As it is well-known, the confocal profiling provides the highest lateral resolution that may be achieved by an optical profiler at least within the FOV of the objective used. This enables to reduce the spatial sampling to $0.10 \mu \mathrm{m}$, which is ideal for the critical dimension measurements required in the present work. We have used the PL $\mu 2300$ profiler from SENSOFAR, which has an extremely high light efficiency for the resolution required. The appropriate selection of both the magnification lens and the numbers of planes required, yield a topography of the sample with a $z$-resolution better than $4 \mathrm{~nm} \mathrm{rms}$. With this device, the roughness parameters $R_{\mathrm{a}}, R_{\mathrm{q}}$ and $R_{\mathrm{z}}$ are automatically calculated.

The results computed by this method for the titanium specimen were $R_{\mathrm{a}}=0.520 \mu \mathrm{m}$ and $R_{\mathrm{q}}=0.671 \mu \mathrm{m}$. Taking into consideration the height precision of confocal microscopy, we could consider this $R_{\mathrm{q}}$ value as a reference for the surface roughness. Bearing in mind that we work with experimental data, all measures are affected by errors. Let $U_{s}\left(\sigma_{h}\right)$ be the systematic uncertainty associated with the experimental arrangement. A possible criterion to discern what lenses are recommendable to carry out the measurements could be that only the values for which the roughness belongs to interval $\sigma_{h}-U_{s} \leq R_{q} \leq \sigma_{h}+U_{s}$ are valid. By analyzing the data of Table 1 with this criterion, we obtain

$$
0.61 \leq R_{q} \leq 0.73 \mu \mathrm{m}
$$


Examining the values obtained, we can conclude that the lenses depicted in the bold box of Table 1 could be rejected to perform the measurements. These ones correspond to lenses of both great focal lengths and diameters.
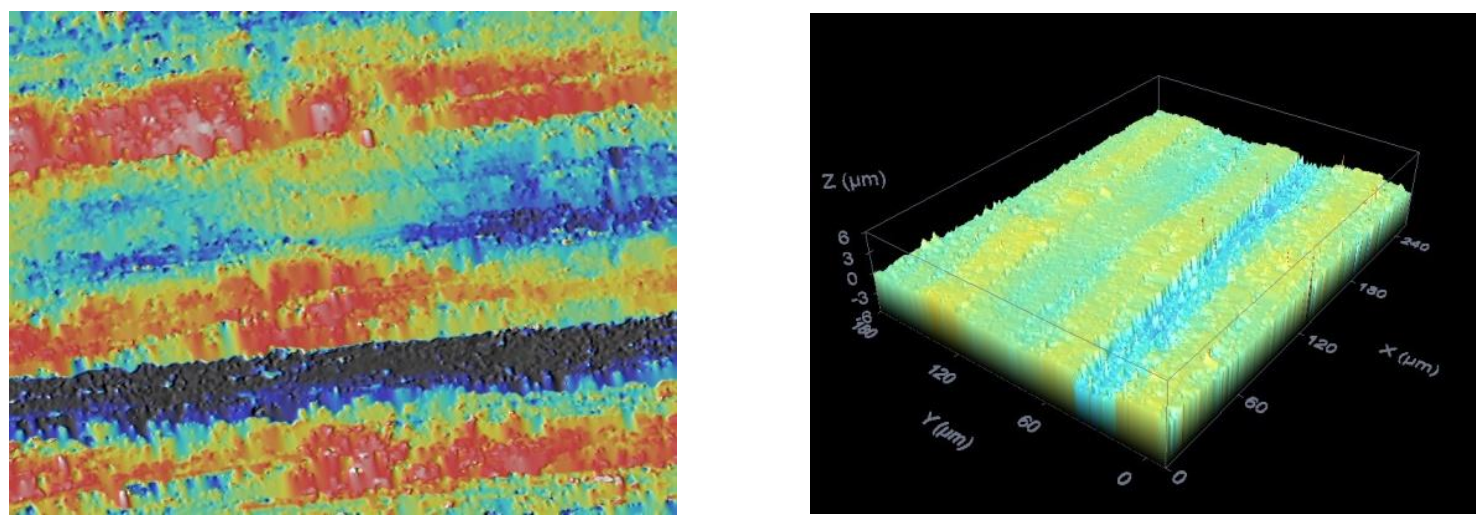

Fig. 3. Titanium surface. (a) 2D diagram. The distinct colors correspond to different microscopic heights.

(b) $3 \mathrm{D}$ representation. Observe the anisotropy.

A detailed analysis of the surface shows anisotropy (Figs 3(a) and 3(b)). This means that the assumption of a Gaussian distribution for the surface heights is not completely correct. However, the results obtained by both techniques are similar within the interval of the proposed criterion.

\subsection{Influence of misalignment}

Due to the different angle of incidence of the beams, the intersecting area of them with the sample surface is also unequal. This fact may produce errors in the measurement process because the speckle recorded by the detector is coming from different regions of the rough surface, leading as a consequence to decorrelation. For that reason, is of great importance to adjust the beam centers.

In order to investigate the influence of a misalignment of their centers, a simple experiment was performed. It consisted in displacing slightly the geometrical center of the first beam with respect to the second one, and thereafter we proceeded the same as explained previously for calculating the roughness.

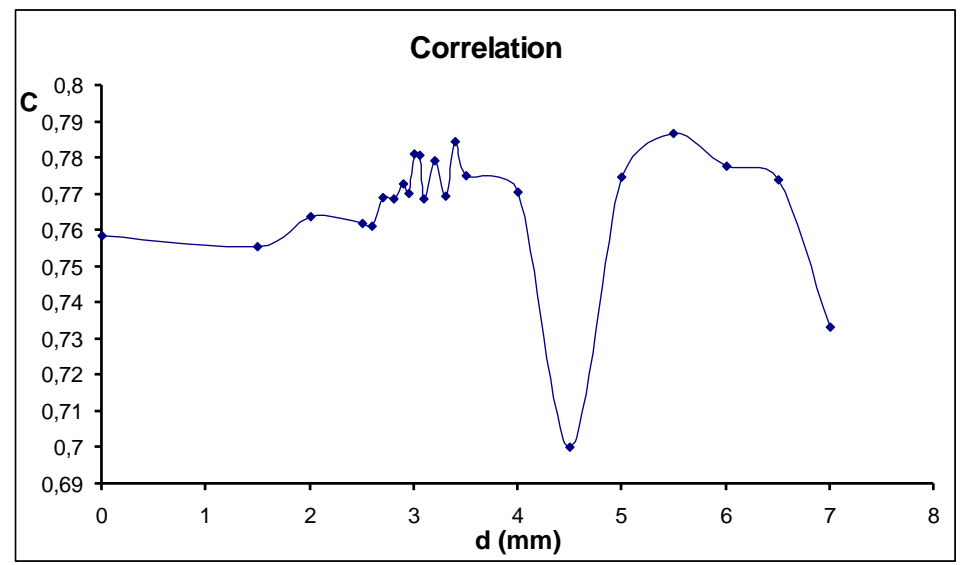

Fig. 4. Correlation curve corresponding to $f=16 \mathrm{~cm}$ and $D=4.3 \mathrm{~cm}$ when misalignment is present. The value of the correlation maximum is 0.78 , which differs from Table 1 for a good adjustment of the optical system. 
Results obtained for the lens of $f=16 \mathrm{~cm}$ and $D=4.3 \mathrm{~cm}$ are represented in Fig. 4. This graph shows the correlation values for different displacements of the CCD camera. The maximum of this curve is 0.78 , which corresponds to a roughness of $1.1 \mu \mathrm{m}$. This value differs considerably from the calculated one when the system is correctly aligned $(\approx 66 \%)$. From this result we can deduce that the correlation degree is extremely sensitive to a misalignment of beams.

To conclude the analysis, we have examined the validity of Eq. (4). From a laboratory point of view, it is important to know the displacement that can be assigned to the camera to obtain maximum correlation. This knowledge can save effort in the development of the experiments. In fact, this would allow moving the camera to a position near the maximum and, from here, to displace again the detector slightly until the maximum of correlation is found. The advantage of this procedure is that it avoids the necessity of performing a large scan as in Fig. 4.

With this in mind, we took all the experimental values of the displacement of the CCD for which a maximum of correlation was obtained. The results are represented in Fig. 5.

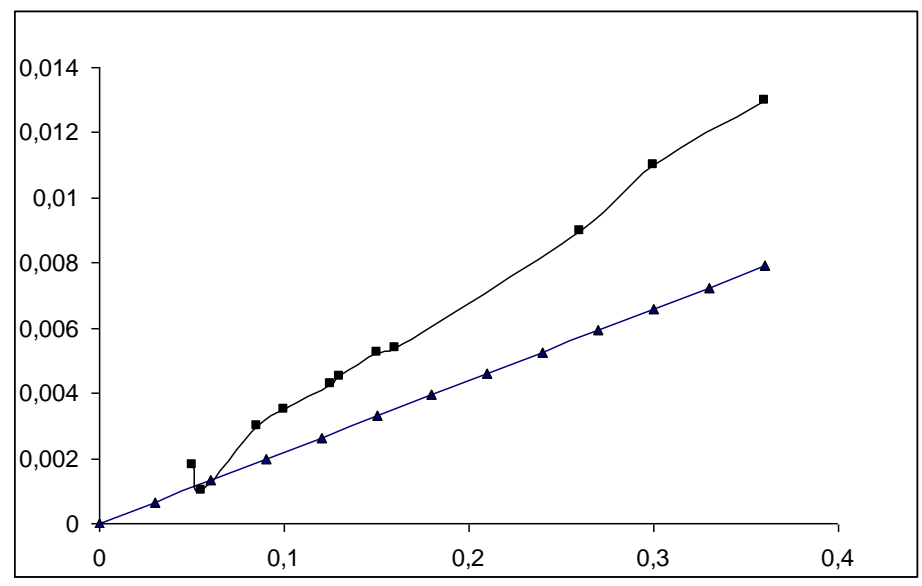

Fig. 5. The lower straight line shows the theoretical curve for the shift of the speckle pattern when the angle of the incident illumination beams is changed (Eq. (4)). The $x$-axis corresponds to the focal length $f$ and the $y$-axis the speckle field displacement on the CCD camera. The upper curve corresponds to the actual displacements for the lenses used in the experiments.

The upper curve corresponds to the measured data and the lower graphic shows the theoretical values obtained applying Eq. (4) directly for all lenses used. The differences are noticeable. Only for lenses of small focal length the theory is in agreement with the experimental results.

From this last result it is inferred that to be sure to match the maximum, it is recommendable to do a scanning with the camera within a large interval, considering Eq. (4) as a first estimate.

\section{Precision of the method}

In this section, the systematic uncertainty of the roughness measurement is calculated. To this end, the basic theory of uncertainties [18] is applied to the expression of $\sigma_{h}$, which adopts the following form:

$$
I_{s}\left(\sigma_{h}\right)=\sum_{j=1}^{N}\left|\frac{\partial \sigma_{h}}{\partial x_{j}}\right| I_{s}\left(x_{j}\right),
$$


where $x_{j}$ are the different variables of the function $\sigma_{h}$. Taking into account that

$$
\sigma_{h}=\frac{\lambda \sqrt{-\ln C}}{4 \pi \delta \alpha \sin \alpha}
$$

applying (6) to this equation, we obtain

$$
I_{s}\left(\sigma_{h}\right)=\left|\frac{\sqrt{-\ln C}}{4 \pi \delta \alpha \sin \alpha}\right| I_{s}(\lambda)+\left|\frac{\cos \alpha \sqrt{-\ln C}}{4 \pi \delta \alpha(\sin \alpha)^{2}}\right| I_{s}(\alpha)+\left|\frac{\lambda \sqrt{-\ln C}}{4 \pi(\delta \alpha)^{2} \sin \alpha}\right| I_{s}(\delta \alpha),
$$

and the relative uncertainty

$$
\frac{I_{s}\left(\sigma_{h}\right)}{\sigma_{h}}=\left|\frac{I_{s}(\lambda)}{\lambda}\right|+\left|\frac{I_{s}(\alpha)}{\operatorname{tg} \alpha}\right|+\left|\frac{I_{s}(\delta \alpha)}{\delta \alpha}\right| .
$$

For our case $I_{s}(\delta \alpha)=1.2 \cdot 10^{-3} \mathrm{rad}$. On the other hand, assuming $I_{s}(\lambda)=0.1 \mathrm{~nm}$ and $I_{s}(\alpha)=1.7 \cdot 10^{-2} \mathrm{rad}$, Eq. (7) yields

$$
\frac{I_{s}\left(\sigma_{h}\right)}{\sigma_{h}}=1.6 \cdot 10^{-4}+1.7 \cdot 10^{-2}+7.4 \cdot 10^{-2} \approx 9.1 \cdot 10^{-2}=9.1 \% \text {. }
$$

It may be observed that the third term contributes more to the uncertainty than the rest. It corresponds to the angular difference between incident beams. To reduce this uncertainty and improve the method, a higher precision of the angle measurement would be necessary.

\section{Conclusions}

By using a sample of titanium, whose roughness was previously measured by a confocal microscope, we have shown that lenses of both great focal lengths and diameters lead to higher roughness values. We also have demonstrated that the misalignment of the incident beams affects the correlation, yielding a very different result as expected. The uncertainty of the method has also been calculated, and this value has proved to be of interest to derive a criterion on how to select a special lens.

\section{References}

[1] Boryczko, A. (2011). Profile irregularities of turned surfaces as a result of machine tool interactions. Metrol. Meas. Syst., 18(4), 691-700.

[2] Wieczorowski, M., Mrozek, R., Andrałojć., P. (2010). The use of surface asperities analysis to investigate wear of bodies in contact on example of brake elements. Metrol. Meas. Syst., 17(2), 271-278.

[3] Twardowski, P. (2011). Surface roughness analysis in milling tungsten carbide with CBN cutters. Metrol. Meas. Syst., 18(1), 105-114.

[4] Murugarajan, A., Samuel, G.L. (2011). Measurement, modelling and evaluation of surface parameter using capacitive-sensor-based measurement system. Metrol. Meas. Syst., 18(3), 403-418.

[5] Zawada-Tomkiewicz, A. (2010). Estimation of surface roughness parameter based on machined surface image. Metrol. Meas. Syst., 17(3), 493-504. 
[6] Goodman, J.W. (1975). Dependence of image speckle contrast on surface roughness. Opt. Commun., 14, 324-327.

[7] Glio, M., Musazzi, S., Perini, U. (1979). Surface measurement by means of speckle wavelength decorrelation. Opt. Commun., 28, 166-170.

[8] Ruffing, B. (1986). Application of speckle-correlation methods to surface-roughness measurement: a theoretical study. J. Opt. Soc. Am. A, 3, 1297-1304.

[9] Pearson, U. (1993). Measurement of surface roughness on rough machined surfaces using spectral speckle correlation and image analysis. Wear, 160, 221-225.

[10] Spagnolo, G.S., Paoletti, D. (1996). Digital speckle correlation for on-line real-time measurement. Opt. Commun., 132, 24-28.

[11] Pérez, Q.F., Rebollo, M.A., Nogert, E.N., Landau, M.R., Gaggioli, N.G. (1996). Relationship between speckle correlation and refraction index variations: applications for roughness measurements. Opt. Eng., $35,1175-1178$.

[12] Lehmann, P., Patzelt, S., Schöne, A. (1997). Surface roughness measurement by means of polychromatic speckle elongation. Appl. Opt., 36, 2188-2197.

[13] Tay, C.J., Toh, S.L., Shang, H.M., Zhang, J. (1995). Whole-field determination of surface roughness by speckle correlation. Appl. Opt., 34, 2324-2335.

[14] Dalmases, F., Cebrián, R., Buendía, M., Romero, C., Salvador, R, Montilla, J. (1988). Speckle correlation technique to determine roughness in the dermatologic interval. Phys. Med. Biol., 33, 913-922.

[15] Yamaguchi, I., Kobayashi, K., Yaroslavsky, L. (2004). Measurement of surface roughness by speckle correlation. Opt. Eng., 43, 2753-2761.

[16] Persson, U. (2006). Surface roughness measurement on machined surfaces using angular speckle correlation. J. Mater. Process. Tech., 180, 233-238.

[17] Ruffing, B. (1987). Non-contacting roughness measurement of technical surfaces by speckle-correlation method. Dissertation, University of Karlsruhe. (in German)

[18] Taylor, J.R. (1982). An introduction to error analysis. Mill Valey, University Science Books. 